\title{
Tibialis Anterior Rupture: A missed diagnosis
}

\author{
by J. Terrence Jose Jerome, MBBS, DNB (Ortho), MNAMS (Ortho) ${ }^{1 凶}$, \\ Mathew Varghese, M.S. (Ortho) ${ }^{2}$, Balu Sankaran, FRCS (C), FAMS ${ }^{3}$, K. Thirumagal, MD $^{4}$
}

The Foot and Ankle Online Journal 3 (9): 2

Closed rupture of the anterior tibial tendon is uncommon. Only a few cases have been reported in the literature. We present a 44 year old man with ruptured tibialis anterior tendon after injury. We discuss both conservative and surgical treatment of tibialis anterior tendon ruptures. Primary surgical repair is indicated in younger, active individuals while older individuals can be treated conservatively by casting in cases of incomplete rupture. It appears that early diagnosis and treatment will provide the best clinical results.

Key words: Tibialis anterior tendon.

Published: September, 2010

This is an Open Access article distributed under the terms of the Creative Commons Attribution License. It permits unrestricted use, distribution, and reproduction in any medium, provided the original work is properly cited. @The Foot and Ankle Online Journal (www.faoj.org)

$\mathrm{T}$ he spontaneous rupture of the tibialis anterior tendon is an uncommon occurrence that most often affects men in their fifth to seventh decades. ${ }^{1}$ Very few cases have been reported in the literature, perhaps because the symptoms are often neglected by the patient as well as by the doctor. ${ }^{11}$ Most often the rupture occurs as a consequence of a sudden plantar flexion of the ankle and pre-existing degenerative changes of the tendon tissue due to systemic disease or iatrogenic local corticoid injections. ${ }^{6,7,10}$ As stated by Bunnell and by McMaster, normal musculotendinous mechanisms may rupture in one of four locations. ${ }^{2,3,4}$ Its order of frequency these are: (1) the insertion (by rupture of the tendons fibers or avulsion of a fragment of bone); (2) the musculotendinous juncture; 5 (3) the muscle belly; (4) the origin (in which rupture is rare).

Address correspondence to:Dr. J. Terrence Jose Jerome, MBBS.,DNB (Ortho), MNAMS (Ortho), FNB (Hand \& Microsurgery)

\footnotetext{
${ }^{1}$ Registrar in Orthopedics, Dept. of Orthopedics, St. Stephen's Hospital, Tiz Hazari, Delhi 54, India.

${ }^{2}$ Head Professor, Department of Orthopedics, St. Stephens Hospital, Tiz Hazari, Delhi, India.

${ }^{3}$ Registrar in Orthopedics, Department of Orthopedics, St. Stephens Hospital, Tiz Hazari, Delhi, India.

${ }^{4}$ Professor Orthopedics, Tamilu,India.
}

Secondary rupture of the tendon may occur from gouty tophaceous deposit within the tendon, psoriasis and sports such as fencing. ${ }^{6,9,13}$ The force and leverage required for rupture are considerable with a large safety factor above the coordinated muscle effort of the individual.

In sharp contrast to this, a tendon showing pathological changes may be ruptured following even slight trauma. This has been graphically demonstrated by Meyer on the shoulder joint and has been repeatedly observed by nearly all orthopaedic surgeons. Burman believed that there was some evidence that the normal anterior tibial tendon could rupture in its substance, but subsequent work has presented sufficient histological and clinical evidence to make this statement doubtful., ${ }^{2,3}$ 


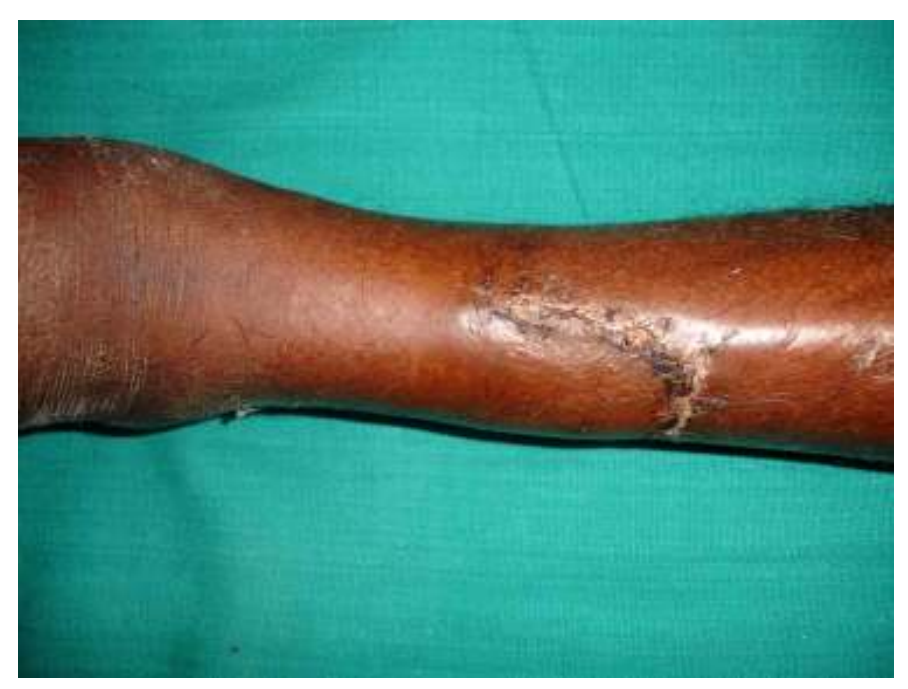

Figure 1 Forty four year-old male post injury to the left leg. There is a laceration repair with missed diagnosis of anterior tibial tendon rupture.

\section{Case Report}

A forty-four year old man presented to our outpatient clinic with history of twisting his left foot while walking downstairs. He appears to have lost his balance and missed his footing resulting in a sudden, sharp pain from the ankle up to the lateral aspect of his leg. He also sustained a laceration in the leg. The injury was followed by pain, slight swelling, and inability to walk in a normal manner.

He underwent primary repair of the laceration and the pain and swelling subsided in two weeks. Sutures were removed and he was referred to our clinic still complaining with an inability to walk. (Fig. 1) On examination he had a typical steppage gait and footdrop. The suture line was healed. The normal contour of the anterior tibial tendon was absent. There was a bulge above the region of the transverse crural ligament. He was unable to dorsiflex and invert the foot. The neurovascular function was intact. Anterior tibial tendon rupture was suspected, so surgery was indicated for primary repair of the tendon.

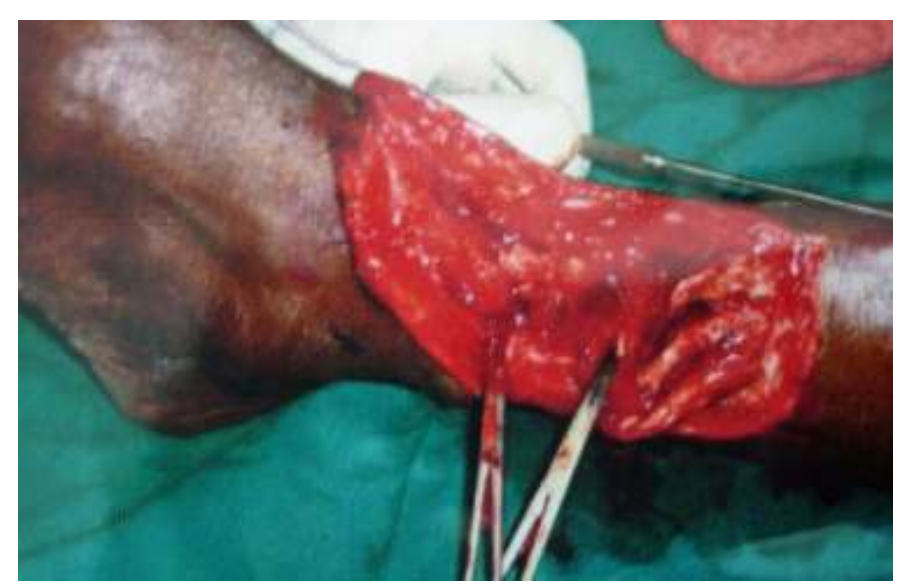

Figure 2 A complete tear of the anterior tibial tendon was noted about 1 inch from its origin.

A curved incision was made distally over the course of the anterior tendon from the cruciate ligament to the insertion. A large granulating mass along the course of the tendon was noted and was found to consist of pseudotendon and injury from the laceration. (Fig. 2) The tear, about one inch from its origin, was complete. After removal of the pseudotendon, the torn ends were approximated and a repair of the tendon was made with 1-0 Prolene using Bunnell technique.

The leg was immobilized in a plaster cast, with the foot at a right angle and in slight inversion. The cast was bivalved three weeks later and daily active assisted exercises were started. At the end of six weeks, active use of the foot with the aid of crutches was permitted, followed in two weeks by free weightbearing. Six months after surgery, there were no subjective complaints and full function, power, and range of motion had been restored near normal to the foot and ankle. (Fig. 3) 


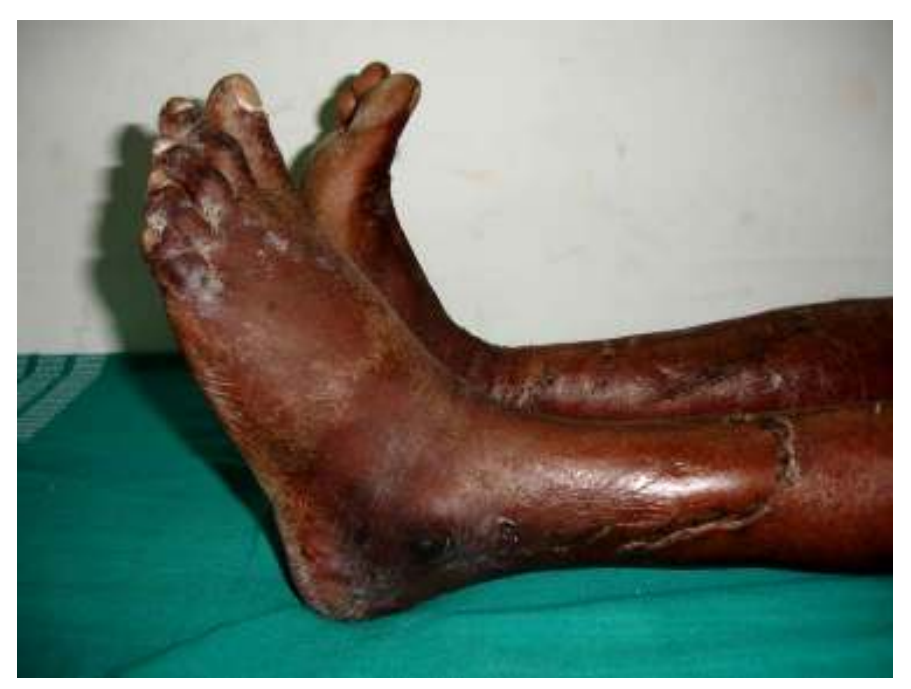

Figure 3 Six months after primary repair, full function and range of motion is appreciated.

\section{Discussion}

The patient may recover sufficiently within half an hour to walk and will notice a lack of coordination, a variable foot-drop or a tendency to stub the toes. In the average patient, adjustment to the injury is progressive. Within a relatively short time, the patient may be able to carry on ordinary activity with little obvious handicap. ${ }^{1-8}$

On physical examination, complete rupture reveals a defect, which is both visible and palpable, between the annular ligament and the insertion of the tibialis anterior tendon. The proximal end may be felt as a bulbous enlargement above or below the annular ligament. In more recent injuries, the usual ecchymoses and tenderness over the site of rupture is usually present. The patient complains of weakness to the foot and some limitations of dorsiflexion. Gait demonstrates a variable degree of foot-drop. Differential diagnoses such as anterior tibial syndrome and peroneal nerve palsy can thus be excluded. $^{11}$ In the chronic cases, the range of dorsiflexion may be reduced by 10 or 15 degrees. The degree of defect and disability may be evident, especially when the patient is asked to walk on his heels. $^{1-9}$
Ultrasound examination and magnetic resonance imaging (MRI) may be helpful. ${ }^{11,12}$ If the ruptured tendon is not seen by ultrasound, MRI will usually confirm the clinical findings. ${ }^{12}$ Complete rupture shows discontinuity of the tendon, thickening of the retracted portion of the tendon, and excess fluid in the tendon sheath. Partial tears show an attenuated tendon with increased surrounding fluid on MRI. Thus, MRI is a useful modality in the definitive detection and characterization of tibialis anterior tendon ruptures. ${ }^{14}$

Conservative treatment has been successful but not complete, especially in cases in which the recognitions of the injury were late and the patient was elderly. However, in patients who respond well to treatment without surgical intervention, there is the possibility that the rupture had been incomplete. One patient operated on by Lapidus was in this category and might have obtained a good functional result under conservative treatment. ${ }^{3}$ Trout, in his series, found those patients who were treated conservatively experienced late sequelae such as foot-drop, mild to moderate flatfoot deformity, and ankle arthrosis. ${ }^{15}$ Thus, high-risk patients can be treated conservatively, but the functional results are less satisfactory. ${ }^{11}$ Low morbidity associated with surgical reconstruction should preclude only the most sedentary of patients from operative treatment. ${ }^{15}$ Markaraian found no statistically significant difference between operative and non-operative treatment in anterior tibialis tendon ruptures. The lack of statistical difference between operative and non-operative groups may be a reflection of the age bimodality noted in the study. Elderly, low demand patients were treated nonsurgically and young active patients were treated operatively. ${ }^{16}$

Operative primary repair is the procedure of choice in younger and middle aged patients with complete and incomplete tears. 'Restitutio ad integrum' can only be achieved by operative treatment and early operative treatment is advantageous. ${ }^{12}$ The surgery requires sufficient exposure along the course of tendon to secure the proximal end, mobilize it, and approximate it to the distal portion. Exposure from the annular ligament to the insertions is usually sufficient. 
Partially incise the dorsum of the tendon tunnel beneath the annular ligament in order to restore a free gliding mechanism. If the tear is found at or near the insertion, the tendon should be fixed to bone as close to its original insertion as possible. If there is a considerable gap, tendon-grafting may be performed by substituting the extensor of the fifth toe or the proximal portion of the tibialis anterior tendons ${ }^{7,10,12,15}$ In using the TA tendon, the tendon is split, starting near the muscle belly, and used as a graft. End-to-end anastomosis and transosseous refixation of the tendon followed by plaster cast for six weeks postoperatively produces good results. ${ }^{11}$

Moberg suggests that in chronic cases, when a marked degree of muscle contracture has developed as a result of prolonged retraction, the diastasis can be bridged by use of the tendon of the extensor hallucis longus or tendon grafts or augmentoplasty. ${ }^{5,7,10,12,15}$ The stump of the proximal portion of the tibialis anterior is sutured to this tendon, which is sectioned with sufficient length to allow it to be brought through the annular ligament and attached to the distal fragment of the tibialis anterior or its insertion. The distal portion of the extensor hallucis tendon is then inserted to the long extensor-of the second toe.

\section{Conclusions}

An appropriate clinical examination including an exact history should lead to the right diagnosis of tibialis anterior tendon rupture. Knowledge of the anatomy in this region may be helpful in the proper diagnosis and interpretation of intra-operative findings. This will aid in choosing the most appropriate surgical procedure. Ruptures can occur in abnormal tendons or in any portion of the tendon and may result in little disability if diagnosed promptly. Early repair does seem to improve the likelihood of a good result. The problem of the contributory effect of atresia, degenerative changes, or both, has not yet been resolved. Primary operative repair, preferably with non absorbable suture, is the treatment of choice for acute ruptures and for patients with high activity levels. Recovery is prompt with little, if any, measurable permanent disability. For chronic ruptures and patients with low demands, conservative management may lead to an equally good outcome.

\section{References}

1. Bernstein RM. Spontaneous rupture of the tibialis anterior tendon. Am J Orthop 1995 24(4):354-356.

2. Burman M. Subcutaneous Rupture of the tendon of the tibialis anticus. Ann Surg 1934 100: 368-372.

3. Lapidus PW. Indirect subcutaneous rupture of the anterior tibial tendon. Report of two cases. Bull Hosp J Dis 1941 2: 119127.

4. McMaster PE. Tendon and muscle ruptures. Clinical and experimental studies on time causes and location of subcutaneous ruptures. J Bone Joint Surg 1933 15: 705-722. 5. Erik MG. Subcutaneous rupture of the tendon of the anterior tibial muscle. Acta Chir Scandinavica 1947 95: 455-469.

6. H. Koenning, C.J. Wirth, U. Wegner Subcutaneous rupture of the tibialis anterior tendon. A case report. Foot and Ankle Surgery 1996; 2 (3), 163-165.

7. Patten A, Wai-Ki P. Spontaneous rupture of the tibialis anterior tendon: A case report and literature review Foot Ankle Int Aug 2000 21(8): 697-700.

8. Kashyap S, Prince R. Spontaneous rupture of the tibialis anterior tendon. Clin Orthop Relat Res1987 (216):159-161. 9. Aydingöz U, Aydingöz O Spontaneous Rupture of the tibialis anterior tendon with psoriasis. Clin Imaging 2002 26(3): 209-211. 10. Kausch T, Rütt J. Spontaneous rupture of the tibialis anterior tendon: Review of the literature and case report. Arch Orthop Trauma Surg 1998 117(4-5): 290-293.

11. Neuber M, Vennemann B, Brug E. Closed rupture of the tendon of the anterior tibial muscle Unfallchirurg 1998 101(4): 319-322.

12. Otte S, Klinger HM, Lorenz F, Haerer T. Operative treatment in case of a closed rupture of the anterior tibial tendon. Arch Orthop Trauma Surg 2002 122(3):188-190. 13. Kelm J, Anagnostakos K, Deubel G, Schliessing P, Schmitt E. The rupture of the tibial anterior tendon in a world-class veteran fencer. Sportverletz Sportschaden. 2004 18(3):148-152. 14. Gallo RA, Kolman BH, Daffner RH, Sciulli RL, Roberts CC, DeMeo PJ. MRI of tibialis anterior tendon rupture. Skeletal Radiol 2004 33(2): 102-106.

15. Trout BM, Hosey G, Wertheimer SJ. Rupture of the tibialis anterior tendon. J Foot Ankle Surg 2000 39(1): 54-58.

16. Markarian GG, Kelikian AS, Brage M, Trainor T, Dias L. Anterior tibialis tendon ruptures: an outcome analysis of operative versus nonoperative treatment. Foot Ankle Int 1998 19(12):792-802. 